\title{
Time and Frequency Varying Noise Floor Estimation for Spectrum Usage Measurement
}

\author{
Hiroki Iwata*, Kenta Umebayashi*, Ahmed Al-Tahmeesschi*, Miguel López-Benítez ${ }^{\dagger}$ and Janne J Lehtomäki ${ }^{\ddagger}$ \\ ${ }^{*}$ Dept. of Electrical and Electronic Engineering, Tokyo University of Agriculture and Technology, Japan \\ ${ }^{\dagger}$ Dept. of Electrical Engineering and Electronics, University of Liverpool, United Kingdom \\ ARIES Research Centre, Antonio de Nebrija University, Spain \\ ${ }^{\ddagger}$ Centre for Wireless Communications, University of Oulu \\ Email: *hiroki_iwata@ieee.org
}

\begin{abstract}
We investigate noise floor (NF) estimation for FFTED (Energy Detection based on FFT)-based spectrum usage measurement in the context of smart spectrum access (SSA), in which spectrum usage information of primary users (PUs), such as channel occupancy rate (COR), will be exploited by secondary users (SUs). In FFT-ED, the NF has to be estimated to set a decision threshold for ED appropriately. In general, the NF is frequency-dependent and its level changes with time leading to the need of estimating the NF regularly while performing the spectrum usage measurement. In this paper, we propose an NF estimation method which exploits prior information regarding the shape of NF and forward consecutive mean excision (FCME) algorithm. Numerical and experimental evaluations show the proposed method enables an accurate NF estimation considering the time and frequency dependencies of the NF. Moreover, we show the proposed method can obtain the almost desired detection performance, but can not the comparative method (the original FCME method).
\end{abstract}

\section{INTRODUCTION}

Due to the fixed spectrum assignment policy and increasing demand for wireless communications, spectrum is a scarce natural resource and there is little room to accommodate new wireless systems. However, several spectrum measurement campaigns around the world have shown that almost all the spectrum is under-utilized in terms of time and space [1]. It means that there is a lot of unused spectrum, which is called white space (WS).

For this issue, dynamic spectrum access (DSA) has been investigated [2]. In DSA, there are primary users (PUs), which have priority regarding spectrum usage, and secondary users (SUs), which can opportunistically access the vacant spectrum as long as the spectrum usage by SUs does not cause any harmful interference to PUs.

In DSA, SUs have to detect WS to protect PUs from the harmful interference and gain the spectrum access opportunities. Moreover, instantaneous information of the target spectrum, either vacant or occupied, is necessary to share the spectrum with PUs whose spectrum usage may change dynamically. To this end, spectrum sensing is an important technique [3]. However, requirements of spectrum sensing, such as accuracy, latency and implementation cost in DSA are substantially high [3].

Not only to resolve the issue of spectrum sensing but also to provide other benefits to DSA, advanced DSA, known as smart spectrum access (SSA), has been investigated [4], [5]. SSA exploits useful statistical information in terms of PU's spectrum usage such as channel occupancy rate (COR). This information can be obtained by long-term, broadband and wide area spectrum usage measurements and it can be used to achieve spectrum sharing smartly. In fact, it has been shown that COR data can enhance spectrum sensing performance [6], [7]. It can also enhance spectrum management, channel selection, MAC protocol for DSA [8], [9].

In this paper, we focus on the spectrum usage measurement part for realizing SSA. In general, the spectrum usage measurement consists of acquisition of the data (e.g., I/Q data), processing the obtained data such as spectrum analysis, spectrum usage detection and estimation of statistical information. Many spectrum usage measurement campaigns have been carried out during the last decade and most of the campaigns utilize frequency-domain energy detection (ED) as a spectrum usage detection method [1].

In the frequency-domain $\mathrm{ED}$, the noise floor (NF) estimation is an important functionality because the detection performance of ED is affected by the accuracy of NF estimation [10]. Erroneous NF estimation can lead to missed detections causing harm to PUs or too many false alarms harming communication by SUs. Most of previous spectrum usage measurements utilizing ED exploited a fixed NF information which is obtained by switching the receiver input to a matched load or is measured in an anechoic chamber before starting the measurements. However, the NF should be estimated periodically due to its time dependency [11]. Moreover, the shape of the NF has frequency dependency and it is equipment-specific [12]. There are several works considering the time dependency of the NF. In [13], the NF estimation method applying forward consecutive mean excision (FCME) algorithm is proposed. It can estimate the NF accurately while carrying out the spectrum usage measurement. However, this method assumes flatness of the noise and thus cannot be directly applied to estimate the frequency-dependent NF.

In this paper, we propose an NF estimation method considering both the time and frequency dependencies of the NF. We assume the NF variation in time and frequency are independent. Under this assumption, the proposed method equalizes the NF in frequency domain by a noise-only ref- 


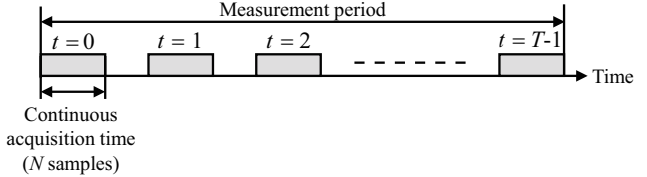

Fig. 1. The configuration of time frames in the measurement process

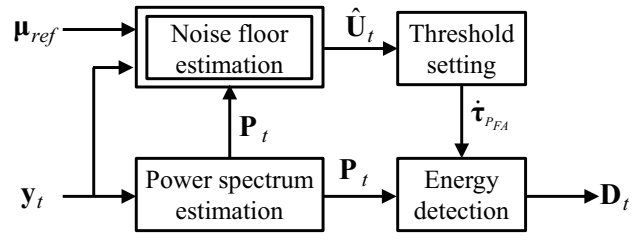

Fig. 2. Block diagram of the spectrum usage measurement process

erence measurement enabling appropriate operation of FCME algorithm. In addition, the method applies the FCME algorithm twice, i.e., along with the axes of time and frequency for increasing efficiency of the NF estimation. Numerical evaluations show the proposed method enables an accurate NF estimation considering the time and frequency dependencies of the NF.

The rest of the paper is organized as follows. In Sect. II, system model and assumptions are described and probabilities of detection and false alarm are analytically derived. A new NF estimation method is proposed in Sect. III. In Sect. IV, the results by means of computer simulation as well as verification experiment are provided. Finally, Sect. V summarizes and concludes this paper.

\section{SYSTEM MODEL AND ASSUMPTIONS}

Configuration of time frames for the spectrum usage measurement is shown in Fig. 1. One continuous spectrum usage measurement (data acquisition) consists of $N$ I/Q baseband samples and is indexed by $t$.

The block diagram of the signal processing used for spectrum usage measurement is shown in Fig. 2. The first process is the power spectrum estimation with Welch FFT [14] using I/Q baseband samples $\mathbf{y}_{t}$. Then, the NF estimation is performed using $\mathbf{y}_{t}$, the power spectrum estimate $\mathbf{P}^{(t)}$ and a reference $\mathrm{NF} \mu_{r e f}$, where we will explain $\mu_{r e f}$ in details in Sect. III. After that, the threshold setting for ED is performed using the estimated NF $\hat{\mathbf{U}}_{t}$. Finally, the ED with the set threshold $\dot{\tau}_{P_{F A}}$ is performed to obtain the spectrum usage decisions $\mathbf{D}_{t}$. Below is the more detailed explanation for the process.

Now let us focus on the $t$ th data acquisition time $(t \in$ $\{0,1, \cdots, T-1\})$. At first, the acquired I/Q baseband signal $\mathbf{y}_{t}=\left[y_{t}[0], y_{t}[2], \cdots, y_{t}[N-1]\right]^{T}$ is divided into $K$ Welch FFT blocks with $N_{s}$ samples. Thus, the I/Q baseband signal $\mathbf{y}_{k}^{(t)}, k \in\{0,1, \cdots, K-1\}$ in the $k$ th Welch FFT block is given by $\mathbf{y}_{k}^{(t)}=\left[y_{t}\left[(k-1) N_{s}+1\right], \cdots, y_{t}\left[(k-1) N_{s}+N_{s}\right]\right]^{T}$. The power spectrum estimation with Welch FFT and ED are performed to obtain spectrum usage decisions for every Welch FFT block.
The power spectrum estimation with Welch FFT consists of three steps: segmentation of $\mathbf{y}_{k}^{(t)}$ with a specific FFT size and an overlap ratio, calculation of multiple power spectra and averaging of the power spectra [14]. The I/Q baseband signal $\mathbf{y}_{k, l}^{(t)}, l \in\{0,1, \cdots, L-1\}$ at $l$ th segment and $k$ th Welch FFT block is given by

$$
\begin{aligned}
& \mathbf{y}_{k, l}^{(t)}=y_{t}[\left.(k-1)(l-1)(1-\rho) N_{F F T}+1\right], \cdots, \\
&\left.y_{t}\left[(k-1)(l-1)(1-\rho) N_{F F T}+N_{F F T}\right]\right]^{T} .
\end{aligned}
$$

where $N_{F F T}$ and $\rho \in[0,1)$ indicate the FFT size and the overlap ratio between the adjacent segments, respectively. In the rest of the paper, we assume $\rho=0.5$ because it has been confirmed to be appropriate choice for good signal detection performance [15]. Moreover, $N_{s}$ and $N_{F F T}$ are assumed to be powers of two. In this case, the number of segments $L$ is given by $L=2 N_{s} / N_{F F T}-1$.

After the segmentation, normal FFT is performed with respect to each segment. The result of FFT operation of $\mathbf{y}_{k, l}^{(t)}$ is given by

$$
\begin{aligned}
\mathbf{Y}_{k, l}^{(t)} & =\frac{1}{\sqrt{N_{F F T}}} \mathbf{F} \mathbf{W} \mathbf{y}_{k, l}^{(t)} \\
& =\left[Y_{k}^{(t)}[l, 0], \cdots, Y_{k}^{(t)}[l, f], \cdots, Y_{k}^{(t)}\left[l, N_{F F T}-1\right]\right]^{T},
\end{aligned}
$$

where $\mathbf{F}=\left(\exp \left(-j 2 \pi m f / N_{F F T}\right)\right)_{m, f=0,1, \cdots, N_{F F T}-1}$ is the discrete Fourier transform matrix. The diagonal matrix $\mathbf{W}=\operatorname{diag}\left(w_{0}, w_{1}, \cdots, w_{N_{F F T}-1}\right)$ is a matrix where its diagonal elements are coefficients $w_{m}$ of the utilized FFT window. It is assumed that $\sum_{m=0}^{N_{F F T}-1} w_{m}^{2}=1$. The type of window function also affects the detection performance [16]. Here, we use Hamming window because it has been shown that it can achieve slightly better performance compared to other window functions in [13].

Finally, power spectrum estimation is performed by averaging the power spectra. Thus, the averaged power spectrum estimate $\mathbf{P}_{k}^{(t)}$ at $k$ th Welch FFT block is given by

$$
\begin{aligned}
\mathbf{P}_{k}^{(t)} & =\frac{1}{L} \sum_{l=0}^{L-1}\left|Y_{k}^{(t)}[l, f]\right|^{2} \\
& =\left[P_{k}^{(t)}[0], \cdots, P_{k}^{(t)}[f], \cdots, P_{k}^{(t)}\left[N_{F F T}-1\right]\right]^{T},
\end{aligned}
$$

where $f=0,1, \cdots, N_{F F T}-1$ indicates the index number of frequency bin. We define a matrix $\mathbf{P}_{t}=\left[\mathbf{P}_{1}^{(t)} \mathbf{P}_{2}^{(t)} \cdots \mathbf{P}_{K}^{(t)}\right]$.

The detection result at the $k$ th Welch FFT block and the $f$ th frequency bin is obtained by the ED as

$$
D_{t}[k, f]=\left\{\begin{array}{l}
1\left(P_{k}^{(t)}[f]>\dot{\tau}_{P_{F A}}\right) \\
0 \text { (otherwise) }
\end{array}\right.
$$

where 1 and 0 correspond to the decisions of occupied spectrum $\left(\mathcal{H}_{1}\right)$ and vacant spectrum $\left(\mathcal{H}_{0}\right)$, respectively. The occupied spectrum $\left(\mathcal{H}_{1}\right)$ indicates that PU signal exists in the frequency bin partially or completely and vacant spectrum $\left(\mathcal{H}_{0}\right)$ indicates otherwise (no signal present). The detection threshold $\dot{\tau}_{P_{F A} A}$ is set based on NF estimate $\hat{\mathbf{U}}_{t}=$ $\left[\hat{U}[t, 0], \hat{U}[t, 1], \cdots, \hat{U}[t, f], \cdots, \hat{U}\left[t, N_{F F T}-1\right]\right]^{T}$ so that $\dot{\tau}_{P_{F A}}$ satisfies constant false alarm rate (CFAR) criterion. 
Threshold setting and NF estimation are explained in Subsect. II-A and Sect. III, respectively.

\section{A. Derivation of Detection and False Alarm Probabilities}

From (2), the result of FFT operation of $\mathbf{y}_{k, l}^{(t)}$ at the $l$ th segment and the $k$ th Welch FFT block is given by

$$
Y_{k}^{(t)}[l, f]= \begin{cases}Z_{k}^{(t)}[l, f] & \left(\mathcal{H}_{0}\right) \\ X_{k}^{(t)}[l, f]+Z_{k}^{(t)}[l, f] & \left(\mathcal{H}_{1}\right),\end{cases}
$$

where $Z_{k}^{(t)}[l, f]$ indicates the result of FFT operation for the noise-only samples $\mathbf{z}_{k, l}^{(t)}$ at $l$ th segment and it is assumed its distribution has a complex Gaussian distribution with mean 0 and variance $U[t, f]$. On the other hand, $X_{k}^{(t)}[l, f]$ indicates the result of FFT operation for the signal samples $\mathbf{x}_{k, l}^{(t)}$ at $l$ th segment and it is assumed its distribution has a complex Gaussian distribution with mean 0 and variance $Q[t, f]$. Moreover, we assume that $Z_{k}^{(t)}[l, f]$ and $X_{k}^{(t)}[l, f]$ are independent of each other and the spectrum occupancy pattern is constant during one Welch FFT block. Then, the averaged spectrum $P_{k}^{(t)}[f]$ at $f$ th frequency bin follows a Gamma distribution with shape parameter $L$ and scale parameter $U[t, f] / L\left(\mathcal{H}_{0}\right)$ or $(U[t, f]+Q[f]) / L\left(\mathcal{H}_{1}\right)^{1}$ and its complimentary cumulative distribution function (CCDF) $F(\tau)$ assuming $x=P_{k}^{(t)}[f]$ is given by

$$
F(\tau)=\int_{\tau}^{\infty} f(x)= \begin{cases}\tilde{\Gamma}\left(L, \frac{\tau}{U[t, f] / L}\right) & : \mathcal{H}_{0} \\ \tilde{\Gamma}\left(L, \frac{\tau}{(U[t, f]+Q[f]) / L}\right) & : \mathcal{H}_{1} .\end{cases}
$$

where $f(x)$ means the probability density function of $x$ $\tilde{\Gamma}(\alpha, \theta)=\frac{1}{\Gamma(\alpha)} \int_{\theta}^{\infty} x^{\alpha-1} \exp (-x) d x$ indicates a normalized incomplete Gamma function and $\Gamma(\cdot)$ is a Gamma function.

Next, we derive the detection threshold satisfying CFAR criterion and the detection and false alarm probabilities. The detection threshold satisfying CFAR criterion $\dot{\tau}_{P_{F A}}$ satisfies the following equation,

$$
\dot{P}_{F A}=\operatorname{Prob}\left(P_{k}^{(t)}[f]>\dot{\tau}_{P_{F A}} \mid \mathcal{H}_{0}\right)=\tilde{\Gamma}\left(L, \frac{\dot{\tau}_{P_{F A}}}{U[t, f] / L}\right),
$$

where $\dot{P}_{F A}$ is a target false alarm probability. Therefore, $\dot{\tau}_{P_{F A}}$ is given by

$$
\dot{\tau}_{P_{F A}}=\frac{U[t, f]}{L} \tilde{\Gamma}^{-1}\left(L, \dot{P}_{F A}\right),
$$

where $\tilde{\Gamma}^{-1}$ indicates the inverse of a normalized incomplete Gamma function. Then, the detection probability is given by

$$
P_{D}=\operatorname{Prob}\left(P_{k}^{(t)}[f]>\dot{\tau}_{P_{F A}} \mid \mathcal{H}_{1}\right)=\tilde{\Gamma}\left(L, \frac{\tilde{\Gamma}^{-1}\left(L, \dot{P}_{F A}\right)}{1+\operatorname{SNR}[f]}\right),
$$

where $\operatorname{SNR}[f]=\frac{Q[f]}{U[f]}$ is the received signal-to-noise ratio (SNR). Eq. (8) indicates an accurate NF information $U[t, f]$ is required to set an appropriate detection threshold.

\footnotetext{
${ }^{1}$ Equivalently, if a random variable $X$ follows a Gamma distribution with shape parameter $L$ and scale parameter $\theta, Y=X / \theta$ follows a Chi-squared distribution with $2 L$ degrees of freedom.
}

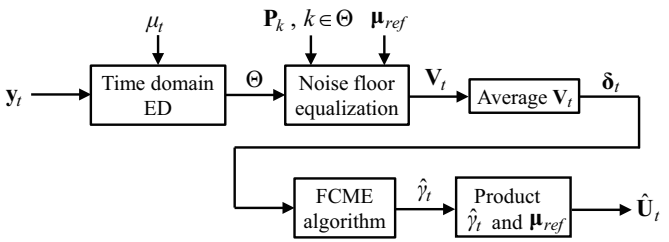

Fig. 3. Block diagram of proposed NF estimation method

\section{NOISE FLOOR ESTIMATION}

\section{A. Time Variation Model of Noise Floor Level}

In this work, we model the NF variation in time as a following relationship

$$
U[j, f]=\gamma_{j} \mu_{\text {ref }}[f],
$$

where $\gamma_{j}$ and $\mu_{\text {ref }}[f]$ indicate the NF level variation factor and the NF at a reference time instant denoted by the reference NF, respectively. This relationship means the the NFs between the reference time instant and any data acquisition times $(t=j)$ does not depend on frequency and its variation is multiplicative

Furthermore, we assume the NF and the noise power $\left(\sigma_{z, t}^{2}\right)$ at least do not change with time during one data acquisition time.

\section{B. Proposed Noise Floor Estimation Method}

In this work, we assume the observation equipment has a radio frequency (RF) terminator to provide noise-only samples. Then, the proposed NF estimation estimates a reference NF $\mu_{\text {ref }}[f]$ based on noise-only samples. The reference NF $\mu_{\text {ref }}[f]$ is calculated by time averaging of power spectrum and it is given by

$$
\mu_{\text {ref }}[f]=\frac{1}{M} \sum_{m=0}^{M-1} P_{m, r e f}[f],
$$

where $M$ and $P_{m, r e f}[f]$ indicate the number of time averaging and power spectrum of noise-only samples, respectively. We define $\boldsymbol{\mu}_{\text {ref }}=\left[\mu_{\text {ref }}[0], \mu_{\text {ref }}[1], \cdots, \mu_{r e f}\left[N_{F F T}-1\right]\right]^{T}$ as a reference NF estimate vector.

Now, our NF estimation problem is equivalent to the estimation of $\gamma_{j}$ given the reference NF $\mu_{r e f}[f]$ while carrying out spectrum usage measurements. The issue in this problem is to estimate $\gamma_{j}$ in an environment where both spectrum occupancy states $\mathcal{H}_{0}$ and $\mathcal{H}_{1}$ exist. $\gamma_{j}$ can be estimated appropriately if the power spectrum samples with state $\mathcal{H}_{0}$ can be identified. In this work, we apply FCME algorithm to discriminate the power spectrum samples with state $\mathcal{H}_{0}$ from the one with state $\mathcal{H}_{1}$.

Block diagram of proposed NF estimation method is shown in Fig. 3. The initial process of proposed method is timedomain ED for I/Q baseband signal $\mathbf{y}_{t}$ to identify the Welch FFT blocks assumed being state $\mathcal{H}_{0}$ across the measurement bandwidth. More specifically, time-domain ED detects state for each Welch FFT block by comparing the energy $T_{k}^{(t)}=$ $\left(\mathbf{y}_{k}^{(t)}\right)^{H} \mathbf{y}_{k}^{(t)}$ for each Welch FFT block with the threshold $\nu_{t}$. 
The set of Welch FFT blocks decided as state $\mathcal{H}_{0}$ by timedomain $\mathrm{ED}, \Theta$ is given as

$$
\Theta=\left\{k \mid T_{k}^{(t)}<\nu_{t}\right\},
$$

where the cardinality of $\Theta$ is defined as $|\Theta|$ and the threshold $\nu_{t}$ is set to satisfy CFAR criterion. The threshold $\nu_{t}$ depends on the noise power $\sigma_{z, t}^{2}$ according to (8) in Subsect. II-A. For this reason, the noise power is estimated by applying FCME algorithm and it is denoted by $\hat{\sigma}_{z, t}^{2}$. The estimation method and threshold setting are explained in Subsect. III-C.

Next step of the proposed method is equalizing process. Thus, the NF is flattened over all frequency bins exploiting the reference NF $\boldsymbol{\mu}_{r e f}$. Specifically, the process is given by

$$
v_{k \in \Theta}^{(t)}[f]=\frac{P_{k \in \Theta}^{(t)}[f]}{\mu_{\text {ref }}[f]} .
$$

As a result, the power spectrum under state $\mathcal{H}_{0}$ has an identical distribution. Thus, the CCDF of $v_{k \in \Theta}^{(t)}[f]$ is given as

$$
F_{v_{k \in \Theta}^{(t)}[f]}(y)= \begin{cases}\tilde{\Gamma}\left(L, \frac{y}{\gamma_{j} / L}\right) & : \mathcal{H}_{0} \\ \tilde{\Gamma}\left(L, \frac{y}{\left(\gamma_{j}+Q[f] / \mu_{r e f}[f]\right) / L}\right) & : \mathcal{H}_{1} .\end{cases}
$$

We define a matrix $\mathbf{V}_{t}$ in which the vectors $\mathbf{v}_{k \in \Theta}^{(t)}$ are stacked along with column, where $\mathbf{v}_{k \in \Theta}^{(t)}=\left[v_{k \in \Theta}^{(t)}[0], \cdots, v_{k \in \Theta}^{(t)}\left[N_{F F T}-1\right]^{T}\right.$.

After that, the proposed method calculates the average of $v_{k \in \Theta}^{(t)}[f]$ denoted as $\delta_{t}[f]$. Thus,

$$
\delta_{t}[f]=\frac{1}{|\Theta|} \sum_{k \in \Theta} v_{k}^{(t)}[f] .
$$

We denote $\boldsymbol{\delta}_{t}$ as $\boldsymbol{\delta}_{t}=\left[\delta_{t}[0], \delta_{t}[1], \cdots, \delta_{t}\left[N_{F F T}-1\right]\right]^{T}=$ $\operatorname{diag}\left(\mathbf{V}_{t} \mathbf{V}_{t}^{T}\right) /|\Theta|$. If the state for the index number of Welch FFT blocks specified in the set $\Theta$ is truly state $\mathcal{H}_{0}$, the mean of $\boldsymbol{\delta}_{t}$ is an appropriate estimate of $\gamma_{j}$. However, $\gamma_{j}$ may be overestimated when the state for the index number of Welch FFT blocks specified in the set $\Theta$ contains the state $\mathcal{H}_{1}$ Therefore, the effect of samples which are affected by power spectrum samples with the state $\mathcal{H}_{1}$ should be excluded before averaging of $\boldsymbol{\delta}_{t}$. Therefore, FCME algorithm is applied to $\delta_{t}$. FCME algorithm identifies the frequency bins in which the state is decided as $\mathcal{H}_{0}$ and then averages $\delta_{t}[f]$ at only frequency bins which are decided as $\mathcal{H}_{0}$. This average value corresponds to the estimate of $\gamma_{j}$ denoted as $\hat{\gamma}_{j}$. The averaged value is calculated as

$$
\hat{\gamma}_{j}=\frac{1}{|\mathrm{C}|} \sum_{f \in \mathrm{C}} \delta_{t}[f],
$$

where the set $\mathrm{C}$ contains the index number of frequency bins which are decided as $\mathcal{H}_{0}$ by FCME algorithm and $|\mathrm{C}|$ indicates the cardinality of $\mathrm{C}$. Specifically, the set $\mathrm{C}$ is given as

$$
\mathrm{C}=\left\{f \mid \delta_{t}[f]<\eta\right\} .
$$

The estimation of $\gamma_{j}$ based on FCME algorithm is explained in Subsect. III-C. by

Finally, the NF estimate by the proposed method is given

$$
\hat{U}[j, f]=\hat{\gamma}_{j} \mu_{\text {ref }}[f] .
$$

\section{Estimation of $\sigma_{z, t}^{2}$ and $\gamma_{t}$ based on FCME algorithm}

FCME algorithm is an iterative method which separates signal samples or noise samples from given samples in an environment that signal samples and noise samples are mixed [17]. Now, let us consider to separate noise samples from power spectrum samples $P[n]$ with $N$ samples exploiting FCME algorithm, where we assume a noise sample follows Gamma distribution with the shape parameter $\alpha$ and the scale parameter $\theta$, where FCME algorithm attempts to estimate $\theta$.

FCME algorithm first sorts the power spectrum samples in an ascending order in terms of power. After that, it calculates the mean of the power spectra using $S$ smallest samples which are assumed to be noise samples. In general, $S=\lceil 0.1 N\rceil$, where $\lceil\cdot\rceil$ is the ceiling function. By assuming that the calculated mean value $\bar{P}$ is correct, the first threshold that attains the target false alarm rate $P_{F A, F C M E}$ such as 0.01 with the calculated mean is obtained based on the assumed distribution of noise samples, which corresponds to Gamma distribution in this paper. Specifically, the threshold $\tau_{\text {FCME }}$ can be set based on (8) in Sect. II-A. Thus,

$$
\tau_{\mathrm{FCME}}=\bar{P} T_{C M E},
$$

where $T_{C M E}=\tilde{\Gamma}^{-1}\left(\alpha, P_{F A, F C M E}\right)$ is a parameter and it can be set in advance [17]. Obviously, the appropriate NF estimate is more than the NF estimate from the first iteration since it is mean of the $S$ smallest samples. Then, the threshold is updated based on the mean value $\bar{P}$ which is calculated by exploiting updated noise samples. The updating of noise samples continues as long as new samples are added from the set of signal samples obtained with the latest threshold.

Now, we describe the estimation method of $\sigma_{z, t}^{2}$ based on FCME algorithm, where $\sigma_{z, t}^{2}$ corresponds to the scale parameter $\theta$ in Gamma distribution. The energy $T_{k}^{(t)}$ for each Welch FFT block under the state $\mathcal{H}_{0}$ can be approximated by Gamma distribution [16]. Thus, the CCDF of $T_{k}^{(t)}$ under the state $\mathcal{H}_{0}$ is given by

$$
F_{T_{k}^{(t)}}(y)=\tilde{\Gamma}\left(\frac{h^{\prime}}{2}, \frac{\left(\frac{2 y}{\sigma_{z, t}^{2}}-c_{1}\right) \sqrt{\frac{h^{\prime}}{c_{2}}}+h^{\prime}}{2}\right),
$$

where $h^{\prime}=c_{2}^{3} / c_{3}^{2}$ and $c_{j}=2 \sum_{i=1}^{N_{s}} \lambda_{i}^{j}$. The symbol $\lambda_{i}$ indicates the $i$ th eigenvalue of the correlation matrix $\mathbf{C}_{z}^{(t)}$ of a noise sample vector $\mathbf{z}_{k}^{(t)}$. Therefore, the threshold for FCME algorithm $\tau_{\mathrm{FCME}}$ has a same form as (19) because both (6) and (20) follow Gamma distribution. Here, $\bar{P}=\frac{1}{N_{s} S} \sum_{k=1}^{S} T^{(t)}(k)$, where $T^{(t)}(k)$ indicates the ordered version of $T_{k}^{(t)}$ and $S$ is the number of Welch FFT blocks satisfying $T_{k}^{(t)}<\tau_{\mathrm{FCME}}$. Moreover, $T_{C M E}=$ $\frac{1}{2}\left\{\sqrt{\frac{c_{2}}{h^{\prime}}}\left(2 \tilde{\Gamma}^{-1}\left(\frac{h^{\prime}}{2}, P_{F A, F C M E}\right)-h^{\prime}\right)+c_{1}\right\}$. Finally, the estimated noise power $\hat{\sigma}_{z, t}^{2}$ corresponds to $\bar{P}$ at the final iteration. 
Table I

PARAMETER SET

\begin{tabular}{|c|c|}
\hline Parameter name & Parameter \\
\hline Welch FFT block size $N_{s}$ & $2^{10}$ \\
\hline FFT size $N_{F F T}$ & $2^{8}$ \\
\hline$M$ & 10000 \\
\hline$\sigma_{z}^{2}$ & $-86 \mathrm{dBm}$ \\
\hline SNR [dB] & {$[-410]$} \\
\hline$\gamma[\mathrm{dB}]$ & -0.5 \\
\hline Measurement bandwidth & $40 \mathrm{MHz}$ \\
\hline Center frequency & $2.437 \mathrm{GHz}$ \\
\hline Signal bandwidth & $20 \mathrm{MHz}$ \\
\hline$\dot{P}_{F A}$ & 0.01 \\
\hline \multicolumn{2}{|c}{} \\
\hline
\end{tabular}

Based on this, the threshold $\nu$ for time-domain ED in the proposed NF estimation is given by

$$
\nu=\hat{\sigma}_{z, t}^{2} T_{C M E} \text {. }
$$

Next, we describe the estimation method of $\gamma_{j}$ based on FCME algorithm, where $\gamma_{j}$ corresponds to the scale parameter $\theta$ in Gamma distribution. In this case, FCME algorithm can also be applied directly because $\delta_{t}[f]$ under the state $\mathcal{H}_{0}$ follows Gamma distribution. Thus, the CCDF of $\delta_{t}[f]$ under the state $\mathcal{H}_{0}$ is given by

$$
F_{\delta_{t}[f]}(y)=\tilde{\Gamma}\left(L, \frac{y}{|\Theta| \gamma_{j} / L}\right),
$$

where the set $\Theta$ is given by (12). Therefore, the threshold for FCME algorithm $\tau_{\mathrm{FCME}}$ has a same form as (19). Here, $\bar{P}=$ $\frac{1}{S} \sum_{f=1}^{S} \delta_{t}(f)$, where $\delta_{t}(f)$ indicates the ordered version of $\delta_{t}[f]$ and $S$ is the number of frequency bins satisfying $\delta_{t}[f]<$ $\tau_{\text {FCME }}$. Moreover, $T_{C M E}=\frac{|\Theta|}{L} \tilde{\Gamma}^{-1}\left(L, P_{F A, F C M E}\right)$. Finally, the estimated NF level variation factor $\hat{\gamma}_{j}$ corresponds to $\bar{P}$ at the final iteration.

\section{NUMERICAL EVALUATIONS}

\section{A. Computer simulations}

In this section, we evaluate the NF estimation performance of the proposed method based on computer simulations as well as experiment. As a comparative method, we apply the original FCME algorithm-based NF estimation method, which does not consider the frequency dependency of the NF. Common parameters are summarized in Table I, where $\sigma_{z}^{2}$ indicates the total noise power in the measurement bandwidth with $40 \mathrm{MHz}$. Figure 4 shows the assumed reference NF in the computer simulations. The true NF corresponds to the reference NF minus $\gamma=0.5$ in $\mathrm{dB}$.

Figure 5 shows RMSE (Root Mean Squared Error) in terms of NF estimate as a function of SNR, where the calculation of the RMSE was performed by MATLAB with 32 digits of precision (using Symbolic Math Toolbox). From this figure, the proposed method can improve the NF estimation performance compared with the FCME method since the proposed method can consider both time and frequency dependencies of

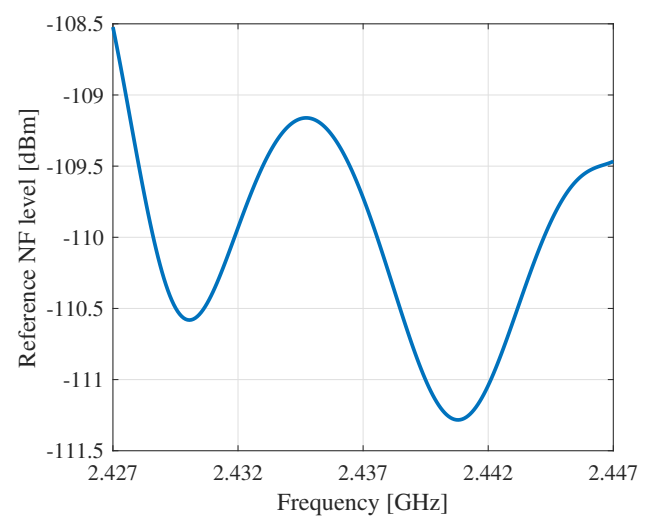

Fig. 4. Assumed reference NF

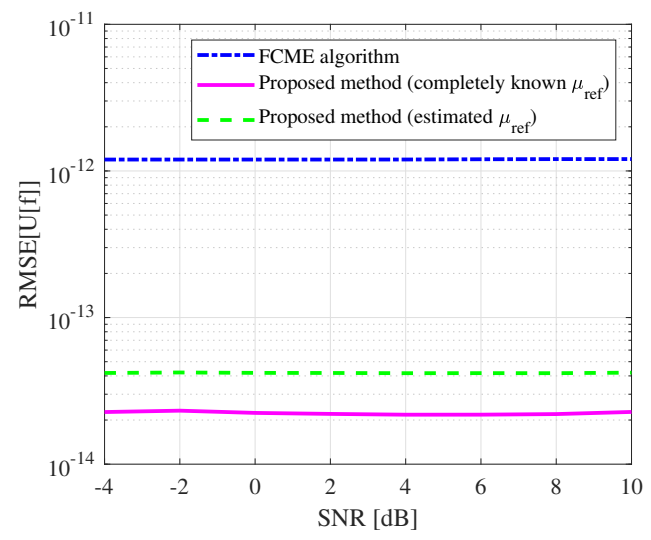

Fig. 5. $\operatorname{RMSE}[U[f]]$ as a function of SNR

the NF. Furthermore, we evaluate the estimation accuracy of the reference NF. As appreciated, the RMSE performance of the proposed method with the known reference NF is better than than the one with the estimated reference NF.

Figure 6 evaluates the RMSE in terms of the detection and false alarm probabilities as a function of SNR. The RMSE means the error between the ideal method and the proposed method or FCME method, where the ideal method knows the true NF and SNR, and the detection and false alarm probabilities can be calculated by Eq. (9) and (7), respectively. From Fig. 6, totally the proposed method has a better RMSE performance than the FCME method. This means the proposed method can achieve accurate NF estimation performance as confirmed in Fig. 5 and set the detection threshold for ED appropriately considering both time and frequency dependencies of the NF. Moreover, the difference of RMSE values between the proposed method with the known reference NF and that with the estimated reference NF is marginal. This indicates the number of time averaging for obtaining the reference NF, $M=10000$ is the appropriate value. 


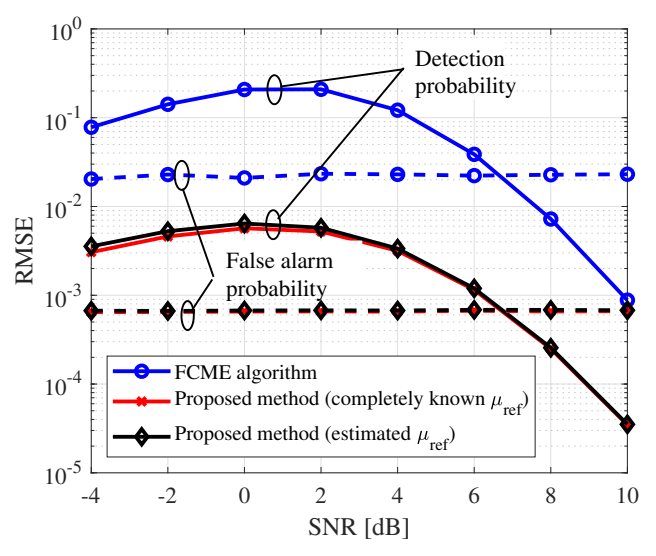

Fig. 6. $P_{D}$ as a function of SNR
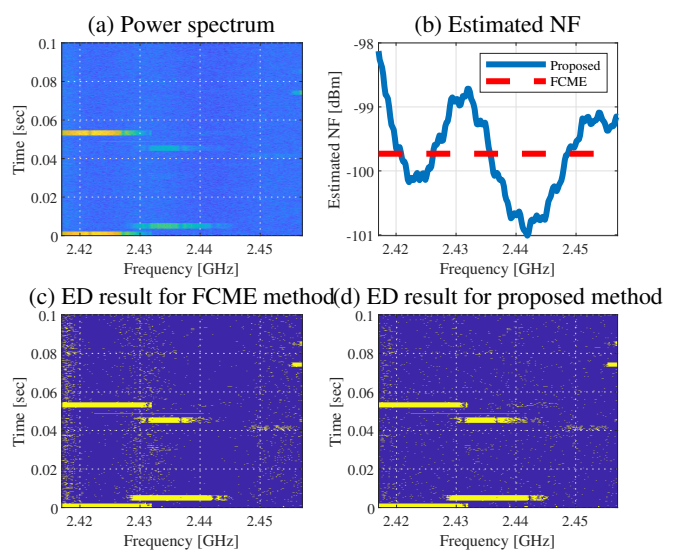

Fig. 7. Experimental results

\section{B. Experiments}

We performed a spectrum usage measurement using a realtime spectrum analyzer (Tektronix RSA306) in the $2.4 \mathrm{GHz}$ WLAN band in our laboratory. Measurement parameters are same as in Table I. Figure 7 shows the experimental results: (a) power spectrum, (b) the estimated NF, (c) ED result for the FCME method and (d) ED result for the proposed method. Comparing the results of (c) and (d) in Fig. 7, there are excessive false alarms in (c), for example the frequencies around $2.41 \mathrm{GHz}$ and $2.43 \mathrm{GHz}$. In fact, Most of the Welch FFT blocks in their frequencies do not contain signal components as confirmed in (a). Thus, the proposed method can estimate the NF properly and this aspect can also be confirmed in (b).

\section{CONCLUSION}

In this work, we propose the NF estimation method considering both the time and frequency dependencies of the NF for spectrum usage measurements. Assuming the NF level variation in time does not depend on frequency, the proposed method equalizes the NF enabling appropriate operation of FCME algorithm in both time and frequency domains. Numerical and experimental evaluations show the proposed method enables an accurate NF estimation considering the frequencydependency and time-varying characteristics of the NF.

\section{ACKNOWLEDGMENT}

This work was supported by the European Commission in the framework of the H2020-EUJ-02-2018 project 5GEnhance (Grant agreement no. 815056) and the Ministry of Internal Affairs and Communications (MIC) of Japan and the JSPS KAKENHI Grant Numbers JP18K04124 and JP18KK0109. The work of M. López-Benítez was supported by British Council under UKIERI DST Thematic Partnerships 2016-17 (ref. DST-198/2017). The work of J. Lehtomäki was supported by the Academy of Finland 6Genesis Flagship (grant no. 318927).

\section{REFERENCES}

[1] M. Höyhtyä, A. Mämmelä, M. Eskola, M. Matinmikko, J. Kalliovaara, J. Ojaniemi, J. Suutala, R. Ekman, R. Bacchus, and D. Roberson, "Spectrum occupancy measurements: A survey and use of interference maps," IEEE Commun. Surveys Tuts, vol. 18, no. 4, pp. 2386-2414, Fourth quarter 2016.

[2] Q. Zhao and B. M. Sadler, "A survey of dynamic spectrum access," IEEE Signal Process. Mag., vol. 24, no. 3, pp. 79-89, May 2007.

[3] T. Yücek and H. Arslan, "A survey of spectrum sensing algorithms for cognitive radio applications," IEEE Commun. Surveys Tuts., vol. 11, no. 1, pp. 116-130, First quarter 2009.

[4] K. Umebayashi, S. Tiiro, and J. J. Lehtomäki, "Development of a measurement system for spectrum awareness," in Proc. IEEE 5GU, Nov. 2014, pp. 234-239.

[5] T. Fujii and K. Umebayashi, "Smart spectrum for future wireless world," IEICE Trans. Commun., vol. E100-B, no. 9, pp. 1661-1673, Sep. 2017.

[6] T. Nguyen, B. L. Mark, and Y. Ephraim, "Spectrum sensing using a hidden bivariate Markov model," IEEE Trans. Wireless Commun., vol. 12, no. 9, pp. 4582-4591, Sept. 2013.

[7] K. Umebayashi, K. Hayashi, and J. J. Lehtomäki, "Threshold-setting for spectrum sensing based on statistical information," IEEE Commun. Lett., Apr. 2017, in press.

[8] M. Wellens, J. Riihijarvi, and P. Mahonen, "Evaluation of adaptive MAC-layer sensing in realistic spectrum occupancy scenarios," in Proc. IEEE DySPAN, Apr. 2010, pp. 1-12.

[9] Y. Xu, A. Anpalagan, Q. Wu, L. Shen, Z. Gao, and J. Wang, "Decisiontheoretic distributed channel selection for opportunistic spectrum access: Strategies, challenges and solutions," IEEE Commun. Surveys Tuts., vol. 15, no. 4, pp. 1689-1713, Fourth quarter 2013.

[10] R. Tandra and A. Sahai, "SNR walls for signal detection," IEEE J. Topics Signal Process., vol. 2, no. 1, pp. 4-17, Feb. 2008.

[11] D. Torrieri, "The radiometer and its practical implementation," in Proc. IEEE MILCOM, Oct. 2010, pp. 304-310.

[12] A. Mariani, A. Giorgetti, and M. Chiani, "Robust detection with lowcomplexity sdrs: A pragmatic approach," in Proc. IEEE PIMRC, Sep. 2018, pp. 1-6.

[13] J. J. Lehtomäki, R. Vuohtoniemi, and K. Umebayashi, "On the measurement of duty cycle and channel occupancy rate," IEEE J. Sel. Areas Commun., vol. 31, no. 11, pp. 2555-2565, Nov. 2013.

[14] P. D. Welch, "The use of fast Fourier transform for the estimation of power spectra: A method based on time averaging over short, modified periodograms," IEEE Trans. Audio Electroacoust., vol. 15, no. 2, pp. 70-73, Jun. 1967.

[15] H. Iwata, K. Umebayashi, S. Tiiro, M. López-Benítez, J. J. Lehtomäki, and Y. Suzuki, "Welch FFT segment size selection method for spectrum awareness system," IEICE Trans. Commun, vol. E99-B, no. 8, pp. 18131823, Aug. 2016.

[16] S. Wang, F. Patenaude, and R. J. Inkol, "Computation of the normalized detection threshold for the FFT filter bank-based summation CFAR detector." Journal of Computers, vol. 2, no. 6, pp. 35-48, 2007.

[17] H. Saarnisaari, P. Henttu, and M. Juntti, "Iterative multidimensional impulse detectors for communications based on the classical diagnostic methods," IEEE Trans. Commun., vol. 53, no. 3, pp. 395-398, Mar. 2005 . 